\title{
Imaging element distribution and speciation in plant cells
}

\author{
Fang-Jie Zhao ${ }^{1,2}$, Katie L. Moore ${ }^{3}$, Enzo Lombi ${ }^{4}$, and Yong-Guan Zhu ${ }^{5,6}$ \\ ${ }^{1}$ National Key Laboratory of Crop Genetics and Germplasm Enhancement and Key Laboratory of Plant Nutrition and Fertilization in \\ Low-Middle Reaches of the Yangtze River, Ministry of Agriculture, College of Resources and Environmental Sciences, Nanjing \\ Agricultural University, Nanjing 210095, China \\ ${ }^{2}$ Rothamsted Research, Harpenden, Hertfordshire AL5 2JQ, UK \\ ${ }^{3}$ Department of Materials, University of Oxford, Oxford OX1 3PH, UK \\ ${ }^{4}$ Centre for Environmental Risk Assessment and Remediation, University of South Australia, Building X, Mawson Lakes Campus, \\ Mawson Lakes, South Australia SA-5095, Australia \\ ${ }^{5}$ Key Laboratory of Urban Environment and Health, Institute of Urban Environment, Chinese Academy of Sciences, Xiamen, China \\ ${ }^{6}$ State Key Laboratory of Urban and Regional Ecology, Research Center for Eco-environmental Sciences, Chinese Academy of \\ Sciences, Beijing 100085, China
}

To maintain cellular homeostasis, concentrations, chemical speciation, and localization of mineral nutrients and toxic trace elements need to be regulated. Imaging the cellular and subcellular localization of elements and measuring their in situ chemical speciation are challenging tasks that can be undertaken using synchrotronbased techniques, such as X-ray fluorescence and X-ray absorption spectrometry, and mass spectrometry-based techniques, such as secondary ion mass spectrometry and laser-ablation inductively coupled plasma mass spectrometry. We review the advantages and limitations of these techniques, and discuss examples of their applications, which have revealed highly heterogeneous distribution patterns of elements in different cell types, often varying in chemical speciation. Combining these techniques with molecular genetic approaches can unravel functions of genes involved in element homeostasis.

\section{Spatial and chemical information of mineral element homeostasis}

Plants take up a range of mineral elements from the soil, some of which are essential for growth, whereas others are non-essential [1]. Deficiencies of essential elements are a major limiting factor for crop production in many areas worldwide, whereas excessive accumulation of both essential and non-essential elements can lead to phytotoxicity [1]. Accumulation of some elements, such as cadmium (Cd) [2] and arsenic (As) [3], in the edible parts of crops may pose a significant risk to human health well before phytotoxicity occurs. By contrast, there is a need to increase essential micronutrients, such as iron (Fe) and zinc $(\mathrm{Zn})$, in plant-

Corresponding authors: Zhao, F.-J. (Fangjie.Zhao@njau.edu.cn); Zhu, Y.-G. (ygzhu@rcees.ac.cn).

Keywords: chemical speciation; plant cells; synchrotron-based techniques.

$1360-1385 / \$$ - see front matter

(C) 2013 Elsevier Ltd. All rights reserved. http://dx.doi.org/10.1016/j.tplants.2013.12.001 based foods to alleviate their deficiencies in humans [4]. Plant nutrition research aims to understand how minerals are acquired, transported, distributed, stored, and used in plants. This knowledge is important not only for sustainable agricultural production but also for ensuring the nutritional quality and safety of agricultural products [5].

Analyses of total elemental concentrations can now be performed using high-throughput platforms to reveal the ionomic profile (see Glossary) of plant tissues [6]. Although the total concentrations of minerals can provide information about the capacity for uptake and translocation, it is well recognized that minerals are distributed heterogeneously across different cell types [7]. Not only may the total concentrations vary at the tissue, cellular, and subcellular scales but also the chemical speciation of minerals may vary. This spatial information is crucial for understanding the homeostasis of minerals, particularly how different cell types and, fundamentally, different genes function in controlling the distribution, complexation, and storage of minerals, and how these processes vary among diverse plant species in the ecophysiological context.

A range of techniques are available for mapping element distribution at various spatial scales. Traditional methods, such as energy-dispersive X-ray microanalysis (EDX) and proton (particle)-induced X-ray emission (PIXE), have been useful in mapping the cellular distributions of macronutrients or metals and metalloids that accumulate to high concentrations in hyperaccumulating plants (e.g., $[8,9]$ ). Visualizing the spatial distribution of micronutrients or toxic trace elements in non-hyperaccumulating plant species is much more challenging because of their low concentrations. Obtaining reliable in situ information about the chemical speciation of mineral elements presents an even greater challenge. These tasks have been greatly facilitated by the novel uses of imaging or analytical techniques that offer greater sensitivity, spatial resolution, or capability for chemical speciation, such as synchrotronbased X-ray absorption or fluorescence and mass spectrometry-based techniques. Here, we review the advantages and limitations of these techniques and discuss examples 


\section{Glossary}

Absorption edge: a sharp discontinuity in the graph of the absorption coefficient of a substance plotted against the wavelength of $X$-rays being absorbed. It represents the minimum energy necessary to free electrons from particular shells of the atoms of interest.

Beamline: the instrumentation that generates and transports synchrotron radiation to an experimental end station where the appropriate radiation is selected, focused, and directed on a sample mounted on a stage. It also includes appropriate detection systems for the signals generated from the sample.

Chemical speciation: the distribution of an element among defined chemical species in a system. Chemical species refer to a specific form of an element defined as electronic or oxidation state, and/or complex or molecular structure. Complexation: formation of a coordination entity consisting of a metal or metalloid center and its ligands.

Ecophysiological: the interrelationship between the physiology of an organism and its environment.

Edge positions: the energy of the incident X-ray where the absorption edge is observed.

Electronegativity: the tendency of an atom or a functional group to attract electrons towards itself.

Energy-dispersive X-ray microanalysis (EDX): a microanalytical technique that uses the characteristic spectrum of X-rays emitted by the specimen after excitation by high-energy electrons to obtain information about its elemental composition. Fast fluorescence detector technologies: devices that are able to detect the fluorescence signal originating from a sample efficiently.

Fourier transformation: a mathematical transformation employed to transform signals between time or spatial domain and frequency domain.

Freeze substitution: the process of replacing ice in a frozen sample with alcohol or another solvent at sub-zero temperatures.

High-pressure freezing: the rapid freezing of a sample under the application of pressure, which allows samples $200-300 \mu \mathrm{m}$ in size to be frozen with minimal formation of damaging ice crystals.

Hyperaccumulating plants (or hyperaccumulators): refer to plant species that are able to accumulate and tolerate large concentrations of metals or metalloids in their aboveground parts. The concentration thresholds used to define hyperaccumulation vary among metals or metalloids, but usually are more than two orders of magnitude higher than those attained by normal nonhyperaccumulating plant species growing on uncontaminated soils.

Image stacks: a dataset where maps of the same areas are collected at different incident energies (e.g., across the absorption edge of an element).

Incident beam: a wave or particle beam which intercepts a sample.

lonomic profile: the feature of the mineral nutrient and trace element composition of an organism.

$\boldsymbol{K}_{\alpha}$ line: refers to the emission line when an electron transitions from a $2 p$ orbital of the second or ' $L$ ' shell to the innermost ' $K$ ' shell.

K-edge: the absorption edge of the $\mathrm{K}$ shell electrons.

Laser ablation system: instrumentation for removing material from a solid surface by irradiating it with a laser beam.

Laser microdissection (LMD) instrument: instrumentation for isolating specific cells of interest from microscopic regions of tissue or organisms using laser beam.

Lateral resolution: the ability of a system to distinguish two points in the direction perpendicular to the direction of an incident beam.

Metalloids: a chemical element that has properties bordering those of metals and non-metals (e.g., arsenic and selenium).

Non-hyperaccumulating plants: plant species that are not hyperaccumulating plants (see above).

Proton (particle)-induced X-ray emission (PIXE): emission of X-rays specific to an element in a specimen when it is irradiated with an ion (proton or $\alpha$ particle) beam, used as a technique to obtain information about the elemental composition of a sample.

Rastering: scanning of a sample in a defined pattern.

Secondary ion mass spectrometry (SIMS): emission of ions from the surface of a sample after bombardment with a high-energy primary ion beam. Secondary ions from the sample are analyzed in a mass spectrometer to build up a chemical map of the sample surface. Isotopes as well as molecular species can be detected.

Spatial resolution: the ability of a system to distinguish the position of two points in a 3D space.

Synchrotron: a particular type of particle accelerator where electrons are accelerated to almost the speed of light to produce electromagnetic radiation (i.e., synchrotron radiation).

Synchrotron-based X-ray absorption: a technique based on the absorption profile of a sample as function of the energy of an incident $X$-ray beam generated at a synchrotron.

Synchrotron X-ray fluorescence (S-XRF): a mapping technique based on the detection of the fluorescence emitted by a sample while it is rastered through an X-ray beam generated at a synchrotron.

Tomographic techniques: techniques that allow the reconstruction of virtual sections within a specimen. of their uses in imaging the distributions of minerals, particularly micronutrients or trace elements, and for probing their chemical speciation in plant cells.

\section{Synchrotron-based techniques}

Synchrotron facilities provide high-intensity photon sources that are $>10$ orders of magnitude brighter than those generated by conventional X-ray tubes [10,11]. Owing to this characteristic, synchrotron-based techniques are highly sensitive and can be used to detect a wide range of elements with a high spatial or lateral resolution. The sensitivity increases with the atomic number, meaning that the techniques are ideally suited to investigations on trace elements and heavy metals or metalloids. Furthermore, these techniques require minimal sample preparation and can be used to probe hydrated plant samples and to investigate element speciation in situ.

There are two main types of synchrotron-based techniques that can be used to analyze plant samples in the context of this review: X-ray fluorescence (XRF, or S-XRF for synchrotron XRF) for imaging elemental distribution and X-ray absorption spectrometry (XAS) for analyzing chemical speciation of elements (Figure 1). Both techniques involve irradiating the sample with X-rays of a definite energy, which excite and eject the core electrons of atoms with a binding energy lower than the incident X-ray. As a result, a vacancy is created in a core orbital, which is then filled by an electron from a higher energy orbital, releasing the excess energy in the form of fluorescence. The energy of this fluorescence signal is characteristic for each element. By rastering a sample through an X-ray beam, fluorescence spectra can be recorded at each position and used to generate XRF elemental maps. A step-by-step guide on the use of S-XRF for elemental mapping in plants is provided in [12]. In an XAS experiment, the energy of the incident X-ray beam is progressively increased while keeping the sample in the same position and the fluorescence signal is collected incrementally. As the energy approaches the binding energy of the core electrons of the element of interest, absorption of the incident beam progressively increases and so does the emitted fluorescence. XAS spectra are therefore generated by detecting and recording the absorption or fluorescence at each energy point. An XAS spectrum is conventionally divided into two parts, the Xray absorption near-edge structure (XANES) and the extended X-ray absorption fine structure (EXAFS) (Figure 1), covering the energy range from approximately -50 to $+200 \mathrm{eV}$ of the absorption edge and from the absorption edge to approximately $+800 \mathrm{eV}$, respectively [10]. XANES is particularly sensitive to the oxidation states of elements and the electronegativity of the ligands, whereas EXAFS can provide information about the coordination chemistry such as the identity and number of the coordinating atoms and the interatomic distance.

In general, there are two types of beamlines used for analyzing elements in plant samples: one uses a millimeter $\mathrm{X}$-ray beam to assess the average speciation of the element of interest in the sample (usually homogenized) and the other uses a micro-focused X-ray beam (tens of nm to a few $\mu \mathrm{m})$ to collect spatial information of intact specimens at the cellular or subcellular scales (Figure 1). 


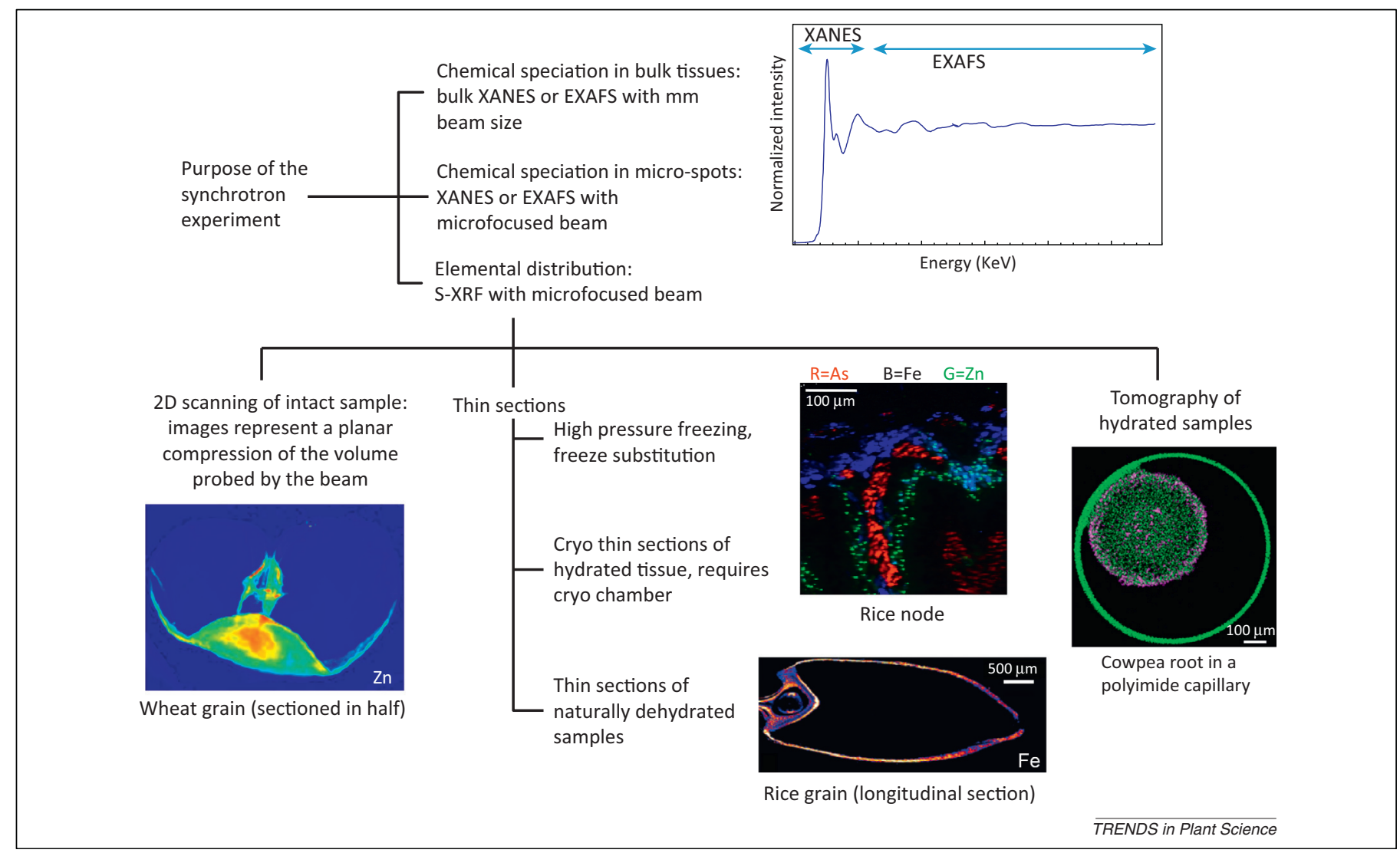

Figure 1. Synchrotron-based techniques for analyzing elemental distribution and speciation in plant tissues. The images of cowpea root, wheat grain, and rice grain are reproduced, with permission, from $[37,75,84]$. The rice node image is based on unpublished data from the authors.

The penetrating nature of X-rays means that 2D elemental maps are a planar compression of the volume probed by the beam. Therefore, elemental maps should be interpreted keeping in mind the morphology of the sample in relation to the variation in thickness and the overlaying tissues. One way to overcome this effect is to use thin sections of uniform thickness (e.g., $[13,14]$ ). Conversely, the penetrating nature of the X-rays enables the use of tomographic techniques to map elements in virtual $2 \mathrm{D}$ sections or in 3D through mathematical reconstructions [15].

The intensity of the X-ray beam means that long exposure may cause damage to the specimen. This has the potential to cause significant artifacts in both element distribution and, perhaps more problematically, chemical speciation. For example, photo-reduction of redox-sensitive elements (e.g., As and Se) may occur during synchrotron beam exposure, causing speciation artifacts [16-18]. It is therefore essential to preserve the sample using cryogenic conditions or preferably by reducing the radiation dose. This can be achieved by using fast detector technologies that drastically reduce the exposure duration and radiation doses [19].

\section{Mass spectrometry-based techniques} Secondary ion mass spectrometry

Secondary ion mass spectrometry (SIMS) is a surfacesensitive technique that uses an energetic primary ion beam to remove particles from the top few atomic layers of a sample surface. The ions emitted during this bombard- ment, known as secondary ions, are analyzed in a mass spectrometer to give information about the elemental or molecular distribution within the sample. The NanoSIMS is a specific type of SIMS instrument that has been designed for high lateral resolution (down to $50 \mathrm{~nm}$ ) imaging while still maintaining high mass resolution (the ability to distinguish between species with nominally the same mass) and high sensitivity ( $\mathrm{mg} / \mathrm{kg}$ range). It is possible to detect most elements in the periodic table, from hydrogen to uranium, as well as their different isotopes. These characteristics offer many advantages for the analysis of elemental distributions in plant cells at the cellular and, crucially, subcellular scale, and enables the use of stable isotope labeling to track element uptake and compartmentalization [20]. The main limitations of this instrument are that it is difficult to analyze some elements, such as $\mathrm{Zn}, \mathrm{Cd}$, and $\mathrm{Mn}$, because of poor secondary ion yield, the difficulty of quantifying actual concentrations, and the inability to obtain chemical state information [21]. The field of view is limited and mapping large areas is relatively slow, although it has become more rapid with the advances in automation and beam control available on newer instruments. Although SIMS is a destructive technique, which can be a disadvantage for some samples, it also enables depth profiling through a sample to build up a $3 \mathrm{D}$ volume.

SIMS must be conducted under an ultra-high vacuum, which imposes limitations on the form of the sample. Fresh tissue cannot be analyzed because of the high water content, instead it must be rapidly cryo-fixed and analyzed 
either frozen-hydrated by cryo-SIMS [20] or, if the instrument does not have a cryo-stage, as is the case for the NanoSIMS, subsequently fixed at low temperature, dehydrated with acetone, and embedded in resin [22]. Sample preparation is the most crucial step in successfully analyzing elemental distributions in plant cells with SIMS analysis [23]. High-pressure freezing followed by freeze substitution has been found to be the best method to preserve cellular and subcellular structures as well as elemental distributions of plant cells for NanoSIMS analysis $[14,22,24]$.

\section{Laser-ablation inductively coupled plasma mass spectrometry}

Laser-ablation inductively coupled plasma mass spectrometry (LA-ICP-MS) can be used to investigate element distribution in solid sample materials by using a focused laser beam in an argon atmosphere under normal pressure. Sample material is ablated by the laser beam and the laser-induced aerosol is transported with a carrier gas ( $\mathrm{He}$ or Ar) into the inductively coupled plasma ion source where ionization takes place. The charged ions are detected by their mass-to-charge ratio, $\mathrm{m} / z$, in the mass spectrometer. The spatial resolution of the common laser ablation systems generally ranges from 50 to $300 \mu \mathrm{m}$ [25]. A high resolution can be achieved using a laser beam with a smaller spot size, although this may lead to insufficient energy focused on the sample and, hence, limited ablation of the material. A laser microdissection (LMD) instrument can substitute a laser ablation system to substantially enhance the spatial resolution [26].

\section{Imaging the cellular and subcellular distribution of elements}

The techniques described above have been used to image element distribution at the tissue and cellular scales of plant samples. Many of the earlier studies focused on hyperaccumulator plants that are able to accumulate large concentrations of metals or metalloids. These studies revealed, for example, $\mathrm{Cd}$ and $\mathrm{Zn}$ accumulation in the trichomes of Arabidopsis halleri [27,28], Zn in the epidermal cells of the leaves of pennycress (Thlaspi praecox) [9], $\mathrm{Ni}$ in the trichomes [29] and the epidermal vacuoles [22] of Alyssum species, Se in the trichomes of milkvetch (Astragalus bisulcatus) [30], As in the vacuoles of Chinese brake fern (Pteris vittata) fronds [31], and $\mathrm{Tl}$ in the veins of candytuft (Iberis intermedia) leaves [32].

More recently, there has been a shift in attention to studying essential or toxic trace elements in non-hyperaccumulator plants: some examples are described below. Fluorescence tomography images obtained at different energies corresponding to different oxidation states of As show a strong accumulation of arsenate $[\mathrm{As}(\mathrm{V})]$ in the iron plaque formed on the root surfaces of aquatic plants such as cattail (Typha latifolia) [33] and rice (Oryza sativa) [34] (Figure 2A), suggesting that iron plaque provides a barrier to the entry of $\mathrm{As}(\mathrm{V})$ into these plants but is less effective in blocking the uptake of arsenite [As(III)], probably owing to a weaker adsorption of As(III) by the plaque [35]. Inside rice roots, $\mathrm{As}(\mathrm{III})$ is preferentially stored in the vacuoles of the pericycle and endodermis cells and is strongly colocalized with $\mathrm{S}$ as revealed by NanoSIMS analysis [24] (Figure 2B). This colocalization is consistent with a vacuolar sequestration of As(III)-thiol (e.g., phytochelatins) complexes. Using S-XRF with a fast detector, the As distribution has been imaged in fresh hydrated roots of cowpea (Vigna unguiculata) following short-term exposure to either As(III) or As(V), showing a strong accumulation of As in the region that is likely to be endodermis and/or pericycle cells [36] (Figure 2C). The same approach has been applied to mapping the in situ distribution of Zn, Cu, Ni, Mn, and Se in hydrated tissues [17,37-39]. A recent study combining S-XRF and NanoSIMS has revealed that As is localized in the vacuoles of the companion cells within the phloem of various types of vascular bundles of the rice node, internode, and leaf sheath [14] (Figure 2D,E). A strong colocalization of As with $\mathrm{S}$ is again evident. Thus, the companion cells possibly provide the most important storage capacity of As in rice aboveground tissues, implying that the biosynthesis of phytochelatins and the vacuolar transport of As(III)-phytochelatin complexes are likely to be much enhanced in this cell type. This distribution pattern also supports the notion that inorganic As is transported to the grain mainly via the phloem [40,41], although with a restricted mobility, presumably because of the vacuolar sequestration. In rice grain, information obtained largely through fluorescence tomography indicates that inorganic As [mainly As(III)] accumulates preferentially in the ovular vascular traces (OVTs) (Figure 2F), which are the conducting tissues transporting nutrients and water to the grain [42-44]. By contrast, methylated As species such as dimethylarsinic acid (DMA) are much more mobile during phloem transport and can permeate into the endosperm of rice more easily than inorganic As $[40,44,45]$.

Mapping the cellular distribution of Cd in non-hyperaccumulator plants is difficult because of its low tissue concentrations and the high energy of its $K_{\alpha}$ line (which is not always accessible at microspectroscopy beamlines). In attempts to overcome these difficulties, researchers have often used artificially high concentrations of Cd that cause severe phytotoxicity and therefore diminish the environmental and physiological relevance of the results obtained (see [46]). Cd has been shown to accumulate in the vascular bundles of roots and leaf trichomes of Arabidopsis thaliana after plants were exposed to a high concentration of $\mathrm{Cd}$ [47]. Using S-XRF, Cd has also been shown to accumulate in the xylem of the enlarged vascular bundles (EVBs) and in the parenchyma cell bridge surrounding the EVBs in the nodes of rice grown in contaminated soil [48]. By contrast, $\mathrm{Zn}$ accumulates preferentially in the parenchyma cell bridge bordering the EVBs and the diffuse vascular bundles (DVBs) [48]; NanoSIMS analysis revealed that Zn was localized to the vacuoles of these cells [14]. Fe and Mn are localized to the fundamental parenchyma cells in the nodes of rice, where there is a strong colocalization of $\mathrm{Fe}$ and $\mathrm{P}$ inside the vacuoles, possibly in insoluble forms [14] (Figure 2D,E), which may explain the antagonistic effect of $\mathrm{P}$ on Fe nutrition [49]. The differences among $\mathrm{Cd}, \mathrm{Zn}, \mathrm{Fe}$, and $\mathrm{Mn}$ distribution in the rice node are striking, even though Cd is known to share some common transporters with the other metals [2]. A possible explanation lies in the different chemical speciation of these metals. 


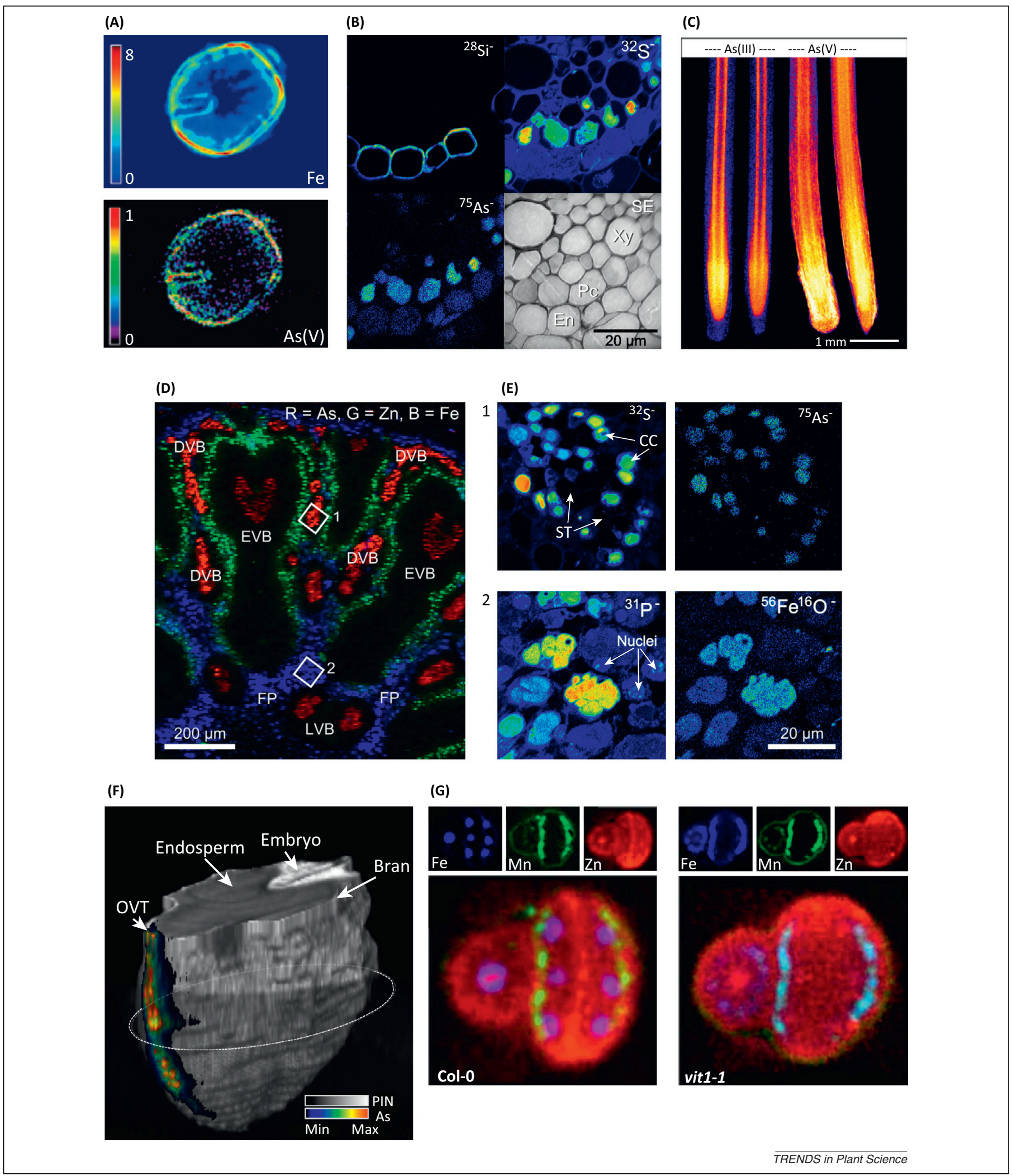

Figure 2. Mapping elemental distribution at the cellular and subcellular scales. (A) Synchrotron X-ray fluorescence (S-XRF) 2D tomography of a rice root growing in an Ascontaminated soil, showing the accumulation of $\mathrm{Fe}$ and $\mathrm{As}(\mathrm{V})$ on the surface iron plaque [34]. (B) NanoSIMS imaging of $\mathrm{Si}, \mathrm{S}$, and $\mathrm{As}$ in a rice root thin section showing colocalization of $\mathrm{As}$ and $\mathrm{S}$ in the vacuoles of pericycle and endodermal cells, and $\mathrm{Si}$ in the apoplast of endodermal cells [24]. Abbreviations: En, endodermis; Pc, pericycle; $\mathrm{SE}$, secondary electron image; $\mathrm{Xy}$, xylem vessel. (C) S-XRF imaging with a fast detector of fresh hydrated roots of cowpea exposed As(V) or As(III) for $24 \mathrm{~h}$ [36]. (D) S-XRF imaging of a rice node thin section showing distinct distribution patterns of As (red), Zn (green), and Fe (blue). Abbreviations: EVB, enlarged vascular bundles; DVB, diffuse vascular bundles; FP, fundamental parenchyma; LVB, large vascular bundles [14]. (E) NanoSIMS imaging of the DVB and FP regions marked as areas 1 and 2 in (D), showing strong colocalization of $A s$ and $S$ in the vacuoles of the companion cells of the phloem in the DVBs and strong colocalization of Fe and $P$ in the vacuoles of FP cells [14]. Abbreviations: CC, companion cells; ST, sieve tubes. (F) S-XRF 3D tomography of a rice grain showing the accumulation of As in the ovular vascular trace (OVT) [42]. (G) SXRF 3D tomography of $\mathrm{Fe}, \mathrm{Mn}$, and $\mathrm{Zn}$ in the seeds of wild type Arabidopsis (Col-0) and the vit1-1 mutant [50]. Reproduced, with permission, from [14,24,34,36,42,50]. 
Mature seeds are ideal for S-XRF or NanoSIMS analysis because they are largely and naturally dehydrated and therefore require minimal sample preparation. Fluorescence tomography has been used to characterize elemental distribution in the seeds of wild type A. thaliana and a mutant defective in the vacuolar iron transporter 1 (VIT1) [50]. Whereas bulk analyses revealed no significant differences in the total $\mathrm{Fe}$ concentrations, the mutant seed exhibited an Fe distribution pattern different to that in the wild type. Iron in wild type seeds is strongly localized to the provascular strands of the hypocotyl, radicle, and cotyledons, probably inside the vacuoles (Figure 2G). By contrast, $\mathrm{Fe}$ is not detected in these cells in the vit1 mutant seeds and is instead located more diffusely in the hypocotyl and radicle, and in the epidermal cells of the cotyledons. This investigation has unraveled the function of VIT1 in vacuolar Fe storage, which is crucial for seedling development after germination. Fluorescence tomography and high-resolution S$\mathrm{XRF}$ has been used to investigate the elemental distribution of wild type Arabidopsis seeds and embryos, and of mutants of endomembrane cation exchangers (CAXs) and overexpression lines [13]. Higher concentrations of Ca were found in the seed coat and in the embryo of the cax mutants compared with that found in the wild type; the mutants also had reduced partitioning of $\mathrm{Ca}$ into organelles and higher levels of $\mathrm{Ca}$ in the cytosol. High resolution $(0.15 \mu \mathrm{m})$ S-XRF analysis of thin sections of embedded embryos showed strikingly different patterns of elemental distribution. In wild type seeds, $\mathrm{Mn}$ is strongly localized to a sub-epidermal layer of cells of the cotyledons, $\mathrm{Fe}$ to the organelles (probably the vacuoles) of the endodermal cells of the radicles and the vasculature of the cotyledons, and $\mathrm{Zn}$ and $\mathrm{Ca}$ are uniformly distributed to all of the cells of the embryo [13]. CAX mutation also alters the distribution of Mn. These examples demonstrate the power of combining stateof-the art imaging techniques with molecular genetic methods to understand the gene functions and cellular homeostasis of elements.

SIMS or NanoSIMS has been used to image a wide range of elements in cereal grains at the cellular and subcellular scales, showing strong localization of minerals with phytate granules in the aleurone cells [51,52], of Se in the protein matrix surrounding the starch granules, of As in the sub-aleurone cells, and in hotspots in the OVT region of the rice grain [45]. Stable isotope ${ }^{70} \mathrm{Zn}$ labeling and LA-ICP-MS has shown that ${ }^{70} \mathrm{Zn}$ strongly accumulates in the crease vascular tissue of wheat (Triticum aestivum) grain, suggesting that the translocation from the maternal to filial tissues may be a bottleneck for $\mathrm{Zn}$ accumulation in the endosperm [53]. For $\mathrm{Fe}$ and $\mathrm{Zn}$ biofortification in cereals, it is important to increase the concentrations of $\mathrm{Fe}$ and $\mathrm{Zn}$ in the endosperm because this is the part that is mostly consumed by humans. Overexpression of nicotianamine synthase and ferritin genes can increase $\mathrm{Fe}$ concentration in the rice endosperm [54]. In LA-ICP-MS analysis, Fe in the endosperm of the transgenic rice appears to accumulate in spots, probably as a consequence of spatially restricted ferritin accumulation [54].

\section{Tracing the movement of elements}

One of the advantages of mass spectrometry-based techniques such as SIMS and LA-ICP-MS is the ability to distinguish and quantify different isotopes, thus enabling stable isotopes to be used as tracers. NanoSIMS has been used to reveal the uptake and competition between rhizosphere microorganisms and plant roots for ${ }^{15} \mathrm{~N}$ labeled ammonium $[55,56]$. Pulsing cut stems of bean (Phaseolus vulgaris) plants with ${ }^{26} \mathrm{Mg},{ }^{41} \mathrm{~K},{ }^{44} \mathrm{Ca}$, and $\mathrm{H}_{2}{ }^{18} \mathrm{O}$ and using cryo-SIMS to image their distribution at different times revealed a rapid exchange of cations between xylem vessels and the adjacent xylem parenchyma cells, and a slow exchange with cambium and phloem; the three cations also exhibited different exchange kinetics [57,58]. In these studies, samples were shock-frozen in melting propane, fractured, and analyzed under cryo-conditions, thus preserving the cellular structure and in situ element distribution.

\section{Probing chemical speciation of elements}

XAS analyses can be performed with bulk samples (usually freeze-dried or, preferably, powdered frozen-hydrated samples) to gain overall information about speciation, or about specific spots of a sample at the micrometer or submicrometer scale using micro-focused XANES and EXAFS. However, owing to the complexity of biological samples, the speciation information obtained is often more accurate with regard to the oxidation state and the type of binding ligands than the exact identities of the ligand molecules. For example, XANES can distinguish As(V), As(III), As(III)-glutathione, and As(III)-phytochelatin, but not the numerous variants of As(III)-phytochelatin or other As(III)-thiol complexes that have been detected in plant tissues based on ex situ extraction and ICP-MS and electrospray mass spectrometry analysis [59]. There are numerous examples of using XAS to investigate the chemical speciation of elements in plant tissues; some examples are described below.

Most plant species appear to be able to reduce $\mathrm{As}(\mathrm{V})$ to $\mathrm{As}$ (III) rapidly, resulting in the dominance of trivalent As inside plant cells [60]. Arsenic is found to be present mainly as As(III) coordinated with thiol compounds in non-hyperaccumulators (e.g., [61-63]), but predominantly as free arsenite or arsenate in As hyperaccumulating ferns (e.g., $[31,64,65])$. A recent study employing the fluorescenceXANES imaging technique showed dramatic changes in the As speciation from the outer to the inner layers of cells of rice and wheat roots that were exposed to $\mathrm{As}(\mathrm{V})$ in the growth medium [66]. As $(\mathrm{V})$ was found only in the rhizodermis, whereas the As(III)-thiol complexes dominated in the cortex and the stele [66]. Confocal XANES is another technique that can be used to target chemical species analysis to specific spatial regions of cells in intact plant tissues [59]. Using this technique, it has been shown that As preferentially accumulated in the epidermal cells of the aquatic plant hornwort (Ceratophyllum demersum) with As(III)-phytochelatins, As(III)-glutathione, and uncomplexed $\mathrm{As}(\mathrm{III})$ and $\mathrm{As}(\mathrm{V})$ as the main chemical species of As [59]. A study has been performed that compared As speciation in rice grain by ex situ extraction followed by high-performance liquid chromatography-ICP-MS 


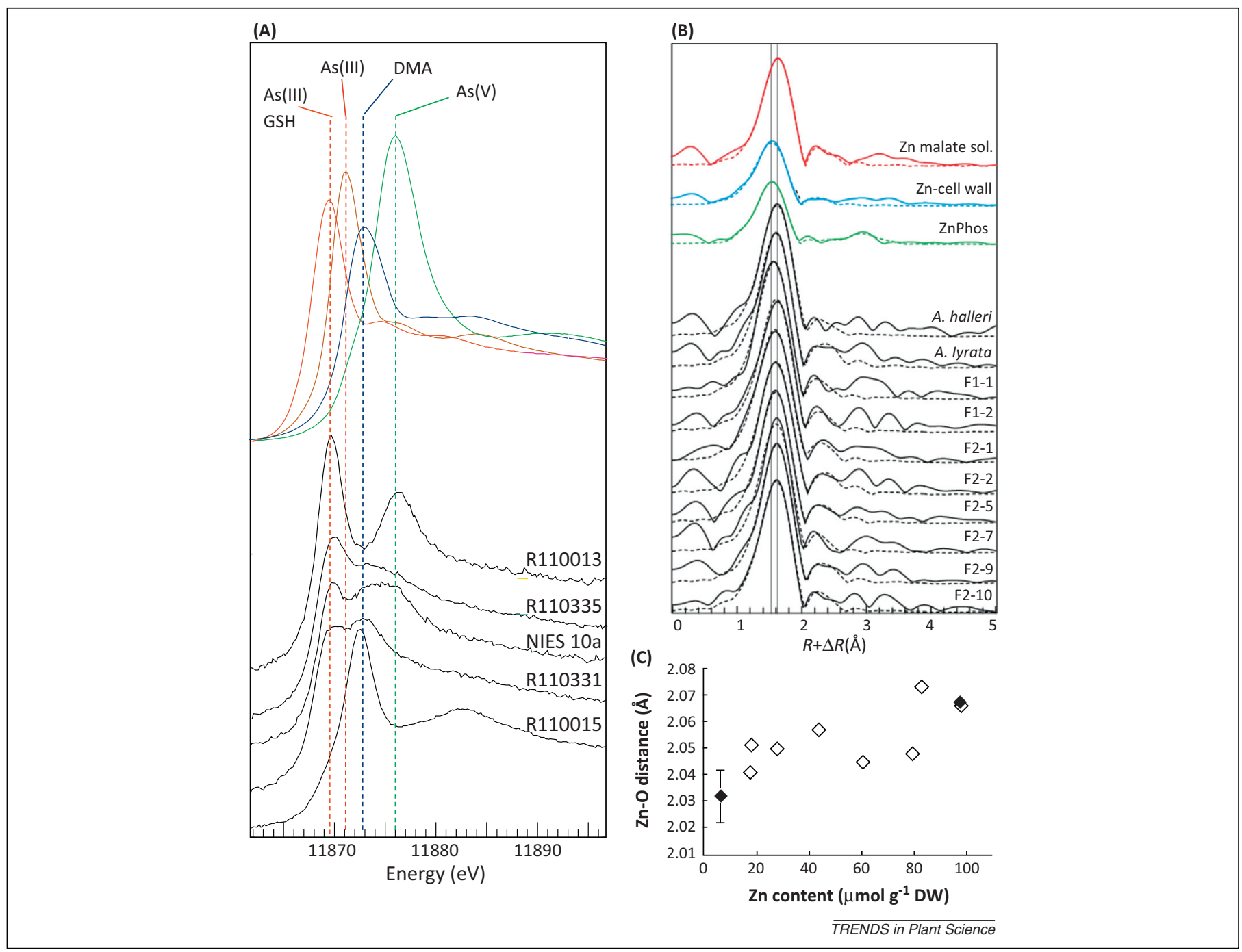

Figure 3. Probing chemical speciation of elements in plant tissues. (A) Synchrotron X-ray absorption near edge structure (XANES) arsenic $K$-edge spectra of arsenic standards and five rice grain samples [67]. Abbreviations: As(III), arsenite; As(III)-GSH, arsenite-glutathione complex; As(V), arsenate; DMA, dimethylarsinic acid. (B) Zinc $K$ edge extended X-ray absorption fine structure (EXAFS) spectra of three Zn standards, Arabidopsis halleri, Arabidopsis lyrata, and their F1 or F2 progeny leaves after Fourier transformation [74]. (C) Average first-shell zinc (Zn)-O distance determined by shell simulations based on the EXAFS spectra in (B) as a function of total Zn concentration [74]. Abbreviation: DW, dry weight. Reproduced, with permission, from $[67,74]$.

analysis and in situ bulk XANES [67] (Figure 3A). The samples had low As concentrations ranging from 0.20 to $0.34 \mu \mathrm{g} / \mathrm{g}$. This study provided evidence of good agreement between the two techniques and demonstrated the high sensitivity of XANES offered by modern synchrotron beamlines. In rice grains, As is present as As(III), As(III)-thiol complexes, As(V), and DMA [43,67].

Cadmium is coordinated mainly with $\mathrm{S}$ (probably phytochelatins or glutathione) in the roots of A. thaliana and mustard (Brassica juncea) [47,68], but with O or N ligands in the trichomes [47]. In the rice node, $\mathrm{Cd}$ is coordinated mainly with $\mathrm{S}$ in the xylem of the enlarged vascular bundles, but with both $\mathrm{S}$ and $\mathrm{O}$ ligands in the phloem [48]. Similar to As hyperaccumulators, Cd hyperaccumulators do not appear to use thiol complexation as the main mechanism to detoxify $\mathrm{Cd}$ given that $\mathrm{Cd}$ is found to be coordinated mainly to $\mathrm{O}$ or $\mathrm{N}$ ligands [69-72].

Depending on plant species, type of tissues and growth conditions, common ligands for binding $\mathrm{Zn}$ in plant cells include organic acids ( $\mathrm{O}$ ligands), histidine, phosphate, or phytate $[28,38,69,73]$. For instance, EXAFS has been used to investigate the speciation of $\mathrm{Zn}$ in parent plants and in the $\mathrm{F}_{1}$ and $\mathrm{F}_{2}$ progenies from crosses between the $\mathrm{Zn}$ hyperaccumulator Arabidopsis halleri and its non-hyperaccumulator relative Arabidopsis lyrata [74]. Figure 3B shows the Zn EXAFS spectra after Fourier transformation of Zn standards and plant samples. Shell simulations were then used to determine the structural parameters for the first and second $\mathrm{Zn}$ coordination shells, which were found to be well fitted with oxygen and carbon as nearest and next nearest atomic neighbors, respectively, as would be expected in $\mathrm{Zn}$-organic acid complexes. The results indicated a correlation between total $\mathrm{Zn}$ in plants and the proportion of $\mathrm{Zn}$ in octahedral coordination (Figure 3C). This is indicative of a dominant complexation by organic acids, where $\mathrm{Zn}$ is sixfold coordinated, in $\mathrm{Zn}$-accumulating plants. In plants that accumulated less Zn, EXAFS analysis revealed a larger proportion of $\mathrm{Zn}$ being fourfold coordinated, probably with the cell wall and phosphate (Figure 3C). In aleurone cells of wheat grain, EXAFS showed that $\mathrm{Fe}$ and $\mathrm{Zn}$ were complexed by phytates; $\mathrm{Fe}$ was coordinated octahedrally by six $\mathrm{O}$ atoms and fewer 
than two $\mathrm{P}$ atoms, whereas $\mathrm{Zn}$ was coordinated tetrahedrally by four $\mathrm{O}$ atoms and approximately $1.5 \mathrm{P}$ atoms [75].

In the case of $\mathrm{Cu}, \mathrm{Cu}(\mathrm{II})$ is reduced to $\mathrm{Cu}(\mathrm{I})$ and coordinated to $\mathrm{S}$, possibly as $\mathrm{Cu}(\mathrm{I})$-metallothionein complexes in tomato (Solanum lycopersicum) and oat (Avena sativa) plants [76] and in alpine pennycress (Noccaea caerulescens; formerly Thlaspi caerulescens), which hyperaccumulates $\mathrm{Zn}$ and $\mathrm{Cd}$ but not $\mathrm{Cu}$ [77]. The coordination chemistry and accessible geometries of $\mathrm{Cu}(\mathrm{I})$-thiolate complexes are highly plastic, a feature that allows $\mathrm{Cu}(\mathrm{I})$ to be bound by various chaperones and delivered to target proteins requiring $\mathrm{Cu}$ [78]. There is also evidence that some $\mathrm{Cu}$ in $N$. caerulescens is bound by nicotianamine or is biomineralized [77]. In contrast to $\mathrm{Cu}$ non-accumulators that rely on $\mathrm{S}$ ligands for complexation, swamp stonecrop (Crassula helmsii), a $\mathrm{Cu}$-accumulating aquatic plant, binds $\mathrm{Cu}$ almost exclusively through O-rich ligands, probably organic acids [79]. Apoplastic binding of $\mathrm{Cu}$ with pectin is also important, particularly in the cell walls of the rhizodermis and the outer cortex of roots [38]. A general pattern that has emerged from comparing metal and metalloid non-accumulators with hyperaccumulators is that strong ligands such as thiol compounds are often used in plant species that are metal or metalloid non-accumulators but are rarely observed in the hyperaccumulators, perhaps because of the cost constraint for the production of such ligands in large quantities.

\section{Concluding remarks and future perspectives}

The cellular status of mineral elements can be defined by three main characteristics: concentration, chemical speciation, and localization; regulation of all three attributes is essential to element homeostasis. The examples described above demonstrate the capabilities of synchrotron and mass spectrometry-based techniques to acquire spatial and chemical information about elements at the cellular or subcellular scales. They reveal the highly heterogeneous distribution patterns of elements in different cell types or subcellular organelles, often varying in chemical speciation. Such information can be used to guide future studies to identify the genes that control element distribution and chemical speciation. Synchrotron-based imaging techniques have already been used to reveal the functions of genes involved in the homeostasis of some nutrients or trace elements $[13,50]$. Owing to the time required for spatial elemental mapping and the availability of beam or machine time, these techniques are suited to studies comparing a limited number of samples (e.g., mutants, overexpressing lines, and their wild type). Screening large arrays of small seeds (e.g., of A. thaliana) for element concentrations is possible using S-XRF [80]; however, it would be difficult to adopt a similar high-throughput approach for other types of plant samples.

As the technologies continue to advance, more novel applications to plant science can be foreseen. For example, chemical speciation at the subcellular scale with synchrotron nanoprobes focusing the beam to $<100 \mathrm{~nm}$ is possible. This would need to be performed on thin cryo-sections of plant samples to avoid changes in speciation due to dehydration and embedding, and to overcome the penetrating nature of the X-rays that can distort the spatial information for geometrically undefined samples. With the advent of fast fluorescence detector technologies, novel approaches that unify S-XRF mapping and XAS speciation are also becoming available. These are based on S-XRF mapping at different energies to obtain speciation rather than elemental maps. This approach in itself is not new given that the distribution of different species of metalloids such as As and Se have been investigated previously by taking advantage of the significant differences in edge positions for different redox states of these elements [31,34,81]. However, with fast detector systems it is now possible to collect image stacks of the same area at many different energies, which has opened the door to speciation mapping for elements having more subtle differences in spectral features between chemical species. From these energy stacks, XANES spectra can then be extracted at each pixel or groups of pixels. This approach, named fluorescence-XANES imaging, has been successfully tested with mineral samples [82] and has also recently been used to analyze plant samples [66].

In the future, although challenging, it may be possible to fit a cryo-stage to the NanoSIMS to avoid complicated sample preparation protocols and ensure minimum elemental redistribution. Further improvements in spatial resolution below $50 \mathrm{~nm}$ will be limited by the ionization process and the collection efficiency of the instrument. For LA-ICP-MS, spatial resolution can be substantially increased, to the nanoscale, with the development of near field-ICP-MS that introduces a tapered probe into the laser radiation field to induce local laser ablation on the sample surface [83]. This technique offers the potential of high resolution and low cost, although the technique has not yet been applied to mapping plant tissues.

\section{Acknowledgments}

F-J.Z. acknowledges support by the Natural Science Foundation of China (grant no. 31372123), the Innovative Research Team Development Plan of the Ministry of Education of China (grant no. IRT1256), and the UK Biotechnology and Biological Sciences Research Council (grant BB/ H006303/1). K.L.M. is supported by the Engineering and Physical Sciences Research Council (EPSRC) grant EP/I026584/1. We thank the Diamond Light Source for the provision of synchrotron beamtime under awards SP6042 and SP7087. E.L. would like to thank the XFM beamline at the Australian Synchrotron for beamtime allocations.

\section{References}

1 Marschner, P. (2012) Marschner's Mineral Nutrition of Higher Plants, Academic Press

2 Clemens, S. et al. (2013) Plant science: the key to preventing slow cadmium poisoning. Trends Plant Sci. 18, 92-99

3 Zhao, F.J. et al. (2010) Arsenic as a food-chain contaminant: mechanisms of plant uptake and metabolism and mitigation strategies. Annu. Rev. Plant Biol. 61, 535-559

4 White, P.J. and Broadley, M.R. (2009) Biofortification of crops with seven mineral elements often lacking in human diets - iron, zinc, copper, calcium, magnesium, selenium and iodine. New Phytol. 182, $49-84$

5 von Wirén, N. (2011) Grand challenges in plant nutrition. Front. Plant Sci. 2,4

6 Salt, D.E. et al. (2008) Ionomics and the study of the plant ionome. Annu. Rev. Plant Biol. 59, 709-733

7 Conn, S. and Gilliham, M. (2010) Comparative physiology of elemental distributions in plants. Ann. Bot. 105, 1081-1102

8 Küpper, H. et al. (1999) Cellular compartmentation of zinc in leaves of the hyperaccumulator Thlaspi caerulescens. Plant Physiol. 119, 305311 
9 Vogel-Mikus, K. et al. (2008) Comparison of essential and non-essential element distribution in leaves of the $\mathrm{Cd} / \mathrm{Zn}$ hyperaccumulator Thlaspi praecox as revealed by micro-PIXE. Plant Cell Environ. 31, 1484-1496

10 Sarret, G. et al. (2013) Use of synchrotron-based techniques to elucidate metal uptake and metabolism in plants. Adv. Agron. 119, 1-82

11 Lombi, E. and Susini, J. (2009) Synchrotron-based techniques for plant and soil science: opportunities, challenges and future perspectives. Plant Soil 320, 1-35

12 Donner, E. et al. (2013) Mapping element distributions in plant tissues using synchrotron X-ray fluorescence techniques. In Plant Mineral Nutrients: Methods and Protocols. Methods in Molecular Biology (Vol. 953) (Maathuis, F.J.M., ed.), pp. 143-159, Springer

13 Punshon, T. et al. (2012) The role of CAX1 and CAX3 in elemental distribution and abundance in Arabidopsis seed. Plant Physiol. 158, $352-362$

14 Moore, K.L. et al. (2014) Combined NanoSIMS and synchrotron X-ray fluorescence reveals distinct cellular and subcellular distribution patterns of trace elements in rice tissues. New Phytol. 201, 104-115

15 de Jonge, M.D. and Vogt, S. (2010) Hard X-ray fluorescence tomography - an emerging tool for structural visualization. Curr. Opin. Struct. Biol. 20, 606-614

16 Lombi, E. et al. (2011) In situ analysis of metal(loid)s in plants: state of the art and artefacts. Environ. Exp. Bot. 72, 3-17

17 Wang, P. et al. (2013) In situ speciation and distribution of toxic selenium in hydrated roots of cowpea. Plant Physiol. 163, 407-418

18 George, G.N. et al. (2012) X-ray-induced photo-chemistry and X-ray absorption spectroscopy of biological samples. J. Synchr. Radiat. 19, 875-886

19 Lombi, E. et al. (2011) Trends in hard X-ray fluorescence mapping: environmental applications in the age of fast detectors. Anal. Bioanal. Chem. 400, 1637-1644

20 Metzner, R. et al. (2008) Imaging nutrient distributions in plant tissue using time-of-flight secondary ion mass spectrometry and scanning electron microscopy. Plant Physiol. 147, 1774-1787

21 Moore, K.L. et al. (2012) Elemental imaging at the nanoscale: NanoSIMS and complementary techniques for element localisation in plants. Anal. Bioanal. Chem. 402, 3263-3273

22 Smart, K.E. et al. (2010) High-resolution elemental localization in vacuolate plant cells by nanoscale secondary ion mass spectrometry. Plant J. 63, 870-879

23 Chandra, S. et al. (2000) Subcellular imaging by dynamic SIMS ion microscopy. Anal. Chem. 72, 104a-114a

24 Moore, K.L. et al. (2011) NanoSIMS analysis reveals contrasting patterns of arsenic and silicon localization in rice roots. Plant Physiol. 156, 913-924

$25 \mathrm{Wu}, \mathrm{B}$. and Becker, J.S. (2012) Imaging techniques for elements and element species in plant science. Metallomics 4, 403-416

26 Becker, J.S. et al. (2010) Scaling down the bioimaging of metals by laser microdissection inductively coupled plasma mass spectrometry (LMDICP-MS). Int. J. Mass Spectrom. 294, 1-6

27 Fukuda, N. et al. (2008) Micro X-ray fluorescence imaging and micro Xray absorption spectroscopy of cadmium hyper-accumulating plant, Arabidopsis halleri ssp gemmifera, using high-energy synchrotron radiation. J. Anal. Atom. Spectrom. 23, 1068-1075

28 Sarret, G. et al. (2002) Forms of zinc accumulated in the hyperaccumulator Arabidopsis halleri. Plant Physiol. 130, 1815-1826

29 McNear, D.H. et al. (2005) Application of quantitative fluorescence and absorption-edge computed microtomography to image metal compartmentalization in Alyssum murale. Environ. Sci. Technol. 39, 2210-2218

30 Freeman, J.L. et al. (2006) Spatial imaging, speciation, and quantification of selenium in the hyperaccumulator plants Astragalus bisulcatus and Stanleya pinnata. Plant Physiol. 142, 124-134

31 Pickering, I.J. et al. (2006) Localizing the biochemical transformations of arsenate in a hyperaccumulating fern. Environ. Sci. Technol. 40, $5010-5014$

32 Scheckel, K.G.et al. (2007) Synchrotron X-ray absorption-edge computed microtomography imaging of thallium compartmentalization in Iberis intermedia. Plant Soil 290, 51-60

33 Blute, N.K. et al. (2004) Arsenic sequestration by ferric iron plaque on cattail roots. Environ. Sci. Technol. 38, 6074-6077

34 Seyfferth, A.L. et al. (2010) Arsenic localization, speciation, and cooccurrence with iron on rice (Oryza sativa L.) roots having variable $\mathrm{Fe}$ coatings. Environ. Sci. Technol. 44, 8108-8113
35 Chen, Z. et al. (2005) Direct evidence showing the effect of root surface iron plaque on arsenite and arsenate uptake into rice (Oryza sativa) roots. New Phytol. 165, 91-97

36 Kopittke, P.M. et al. (2012) Examination of the distribution of arsenic in hydrated and fresh cowpea roots using two- and three-dimensional techniques. Plant Physiol. 159, 1149-1158

37 Lombi, E. et al. (2011) Fast X-ray fluorescence microtomography of hydrated biological samples. PLoS ONE 6, e20626

38 Kopittke, P.M. et al. (2011) In situ distribution and speciation of toxic copper, nickel, and zinc in hydrated roots of cowpea. Plant Physiol. 156, $663-673$

39 Kopittke, P.M. et al. (2013) Distribution and speciation of Mn in hydrated roots of cowpea at levels inhibiting root growth. Physiol. Plant. 147, 453-464

40 Carey, A.M. et al. (2010) Grain unloading of arsenic species in rice. Plant Physiol. 152, 309-319

41 Zhao, F.J. et al. (2012) Arsenic translocation in rice investigated using radioactive ${ }^{73}$ As tracer. Plant Soil $350,413-420$

42 Carey, A.M. et al. (2011) Phloem transport of arsenic species from flag leaf to grain during grain filling. New Phytol. 192, 87-98

43 Lombi, E. et al. (2009) Speciation and distribution of arsenic and localization of nutrients in rice grains. New Phytol. 184, 193-201

44 Zheng, M.Z. et al. (2013) Differential toxicity and accumulation of inorganic and methylated arsenic in rice. Plant Soil 365, 227-238

45 Moore, K.L. et al. (2010) NanoSIMS analysis of arsenic and selenium in cereal grain. New Phytol. 185, 434-445

46 Van Belleghem, F. et al. (2007) Subcellular localization of cadmium in roots and leaves of Arabidopsis thaliana. New Phytol. 173, 495-508

47 Isaure, M.P. et al. (2006) Localization and chemical forms of cadmium in plant samples by combining analytical electron microscopy and Xray spectromicroscopy. Spectrochim. Acta B: Atom. Spectrom. 61, $1242-1252$

48 Yamaguchi, N. et al. (2012) Role of the node in controlling traffic of cadmium, zinc, and manganese in rice. J. Exp. Bot. 63, 2729-2737

49 Zheng, L.Q. et al. (2009) Physiological and transcriptome analysis of iron and phosphorus interaction in rice seedlings. Plant Physiol. 151, 262-274

$50 \mathrm{Kim}$, S.A. et al. (2006) Localization of iron in Arabidopsis seed requires the vacuolar membrane transporter VIT1. Science 314, 1295-1298

51 Heard, P.J. et al. (2002) Determination of the elemental composition of mature wheat grain using a modified secondary ion mass spectrometer (SIMS). Plant J. 30, 237-245

52 Moore, K.L. et al. (2012) Localisation of iron in wheat grain using high resolution secondary ion mass spectrometry. J. Cereal Sci. 55, 183-187

53 Wang, Y.X. et al. (2011) Stable isotope labelling and zinc distribution in grains studied by laser ablation ICP-MS in an ear culture system reveals zinc transport barriers during grain filling in wheat. New Phytol. 189, 428-437

54 Wirth, J. et al. (2009) Rice endosperm iron biofortification by targeted and synergistic action of nicotianamine synthase and ferritin. Plant Biotechnol. J. 7, 631-644

55 Clode, P.L. et al. (2009) In situ mapping of nutrient uptake in the rhizosphere using nanoscale secondary ion mass spectrometry. Plant Physiol. 151, 1751-1757

56 Jones, D.L. et al. (2013) Competition between plant and bacterial cells at the microscale regulates the dynamics of nitrogen acquisition in wheat (Triticum aestivum). New Phytol. 200, 796-807

57 Metzner, R. et al. (2010) Tracing cationic nutrients from xylem into stem tissue of French bean by stable isotope tracers and cryo-secondary ion mass spectrometry. Plant Physiol. 152, 1030-1043

58 Metzner, R. et al. (2010) Contrasting dynamics of water and mineral nutrients in stems shown by stable isotope tracers and cryo-SIMS. Plant Cell Environ. 33, 1393-1407

59 Mishra, S. et al. (2013) Speciation and distribution of arsenic in the nonhyperaccumulator macrophyte Ceratophyllum demersum. Plant Physiol. 163, 1396-1408

60 Zhao, F.J. et al. (2009) Arsenic uptake and metabolism in plants. New Phytol. 181, 777-794

61 Pickering, I.J. et al. (2000) Reduction and coordination of arsenic in Indian mustard. Plant Physiol. 122, 1171-1177

62 Bluemlein, K. et al. (2008) Can we trust mass spectrometry for determination of arsenic peptides in plants: comparison of LC-ICP- 
MS and LC-ES-MS/ICP-MS with XANES/EXAFS in analysis of Thunbergia alata. Anal. Bioanal. Chem. 390, 1739-1751

63 Castillo-Michel, H. et al. (2011) Localization and speciation of arsenic in soil and desert plant Parkinsonia florida using mu XRF and mu XANES. Environ. Sci. Technol. 45, 7848-7854

64 Webb, S.M. et al. (2003) XAS speciation of arsenic in a hyperaccumulating fern. Environ. Sci. Technol. 37, 754-760

65 Lombi, E. et al. (2002) Arsenic distribution and speciation in the fronds of the hyperaccumulator Pteris vittata. New Phytol. 156, 195-203

66 Kopittke, P.M. et al. (2013) Laterally resolved speciation of arsenic in roots of wheat and rice using fluorescence-XANES imaging. New Phytol. http://dx.doi.org/10.1111/nph.12595

67 Maher, W. et al. (2013) Measurement of inorganic arsenic species in rice after nitric acid extraction by HPLC-ICPMS: verification using XANES. Environ. Sci. Technol. 47, 5821-5827

68 Salt, D.E. et al. (1995) Mechanisms of cadmium mobility and accumulation in Indian mustard. Plant Physiol. 109, 1427-1433

69 Küpper, H. et al. (2004) Tissue- and age-dependent differences in the complexation of cadmium and zinc in the cadmium/zinc hyperaccumulator Thlaspi caerulescens (Ganges ecotype) revealed by X-ray absorption spectroscopy. Plant Physiol. 134, 748-757

70 Huguet, S. et al. (2012) Cd speciation and localization in the hyperaccumulator Arabidopsis halleri. Environ. Exp. Bot. 82, 54-65

71 Tian, S.K. et al. (2011) Cellular sequestration of cadmium in the hyperaccumulator plant species Sedum alfredii. Plant Physiol. 157, 1914-1925

72 Vogel-Mikus, K. et al. (2010) Complexation of cadmium in seeds and vegetative tissues of the cadmium hyperaccumulator Thlaspi praecox as studied by X-ray absorption spectroscopy. Plant Soil 331, 439-451

73 Salt, D.E. et al. (1999) Zinc ligands in the metal hyperaccumulator Thlaspi caerulescens as determined using X-ray absorption spectroscopy. Environ. Sci. Technol. 33, 713-717

74 Sarret, G. et al. (2009) Zinc distribution and speciation in Arabidopsis halleri $\times$ Arabidopsis lyrata progenies presenting various zinc accumulation capacities. New Phytol. 184, 581-595
75 Neal, A.L. et al. (2013) Iron and zinc complexation in wild-type and ferritin-expressing wheat grain: implications for mineral transport into developing grain. J. Biol. Inorg. Chem. 18, 557-570

76 Ryan, B.M. et al. (2013) Copper speciation and isotopic fractionation in plants: uptake and translocation mechanisms. New Phytol. 199, 367378

77 Mijovilovich, A. et al. (2009) Complexation and toxicity of copper in higher plants. II. Different mechanisms for copper versus cadmium detoxification in the copper-sensitive cadmium/zinc hyperaccumulator Thlaspi caerulescens (Ganges ecotype). Plant Physiol. 151, 715-731

78 Pushie, M.J. et al. (2012) The fictile coordination chemistry of cuprousthiolate sites in copper chaperones. Biochim. Biophys. Acta Bioenerg. 1817, 938-947

79 Küpper, H. et al. (2009) Complexation and toxicity of copper in higher plants. I. Characterization of copper accumulation, speciation, and toxicity in Crassula helmsii as a new copper accumulator. Plant Physiol. 151, 702-714

80 Young, L.W. et al. (2006) A high-throughput determination of metal concentrations in whole intact Arabidopsis thaliana seeds using synchrotron-based X-ray fluorescence spectroscopy. J. Synchr. Radiat. 13, 304-313

81 Pickering, I.J. et al. (2000) Quantitative, chemically specific imaging of selenium transformation in plants. Proc. Natl. Acad. Sci. U.S.A. 97, 10717-10722

82 Etschmann, B.E. et al. (2010) Reduced As components in highly oxidized environments: evidence from full spectral XANES imaging using the Maia massively parallel detector. Am. Mineral. $95,884-887$

$83 \mathrm{Wu}$, B. and Becker, J.S. (2011) Imaging of elements and molecules in biological tissues and cells in the low-micrometer and nanometer range. Int. J. Mass Spectrom. 307, 112-122

84 Johnson, A.A.T. et al. (2011) Constitutive overexpression of the OsNAS gene family reveals single-gene strategies for effective iron- and zincbiofortification of rice endosperm. PLoS ONE 6, e24476

\title{
Plant Science Conferences in 2014
}

\author{
GRC: Plant Molecular Biology \\ Decision-Making Pathways, Networks, and Models in Plant Biology \\ 20-25 July, 2014 \\ Holderness, USA \\ http://www.grc.org/programs.aspx?year=2014\&program=plantmolec
}

2014 Wisconsin Plant Proteomics Workshop

21-25 July, 2014

Madison, USA

http://www.biotech.wisc.edu/ppw

EMBO Workshop: Intercellular Communication in Plant Development and Disease

24-29 August, 2014

Bischoffsheim, France

http://events.embo.org/coming-soon/index.php?EventID=w14-11

25th International Conference on Arabidopsis Research (ICAR 2014)

28 July-1 August, 2014

Vancouver, Canada

http://arabidopsisconference2014.org/ 